\title{
Electrochemical Analysis of Cobalt Hydroxide Layered Carbon Spheres Synthesized Via Hydrothermal Route
}

\author{
C. Sambathkumar, R. Ranjithkumar, S. Ezhil Arasi, A. Arivarasan, P. Devendran
}

\begin{abstract}
Cobalt hydroxide layered carbon spheres was prepared via hydrothermal method. The structure of prepared carbon sphere and cobalt hydroxide was analyzed using powder $x$-ray diffraction analysis. Fourier transform infrared spectroscopic analysis helped in analyzing functional groups of prepared nanomaterials. The surface morphology, elemental composition and purity of cobalt hydroxide layered carbon sphere were investigated through scanning electron microscopy, energy dispersive spectroscopic technique equipped with mapping analysis. The redox behavior, charge discharge property was carried out using cyclic voltammetry and galvanostatic charge-discharge studies.
\end{abstract}

Keywords-Cobalt hydroxide, carbon sphere, hydrothermal, SEM, cyclic voltammetry, charge-discharge.

\section{INTRODUCTION}

$\mathrm{C}$ increase the efficiency of the electrode used in electrochemical devices. Researchers are keen in synthesizing the materials based on the morphology, as the morphology of materials changes the properties of prepared nanomaterials [1-3]. This is in agreement with the surface to volume ratio of the nanomaterials. Along with this, there are various carbon derivatives such as carbon nanotubes, carbon fibres, graphite, graphene oxide, are available, among those the carbon sphere is specific for their morphology $[4,5]$.

Metal oxide, carbides, nitrides and hydroxides are found to be the most commonly used electrode materials. On comparing metal oxide, metal hydroxides are efficient in their physical, optical and chemical properties etc. Transition metal hydroxides have wide range of applications such as catalysis, dye degradation, electrochemical storage and conversion

Revised Manuscript Received on December 16, 2019.

* Correspondence Author

P. Devendran, Department of Physics, International Research Centre,Kalasalingam Academy of Research and Education,

Krishnankoil-626126.Tamil Nadu, India. p.devendran@klu.ac.in, pdevavenmani@gmail.com

C. Sambathkumar, Department of Physics, International Research Centre,Kalasalingam Academy of Research and Education, Krishnankoil-626126.Tamil Nadu, India.

R. Ranjithkumar, Department of Physics, International Research Centre,Kalasalingam Academy of Research and Education, Krishnankoil-626126.Tamil Nadu, India.

S. Ezhil Arasi, Department of Physics, International Research Centre,Kalasalingam Academy of Research and Education, Krishnankoil-626126.Tamil Nadu, India.

A. Arivarasan, Department of Physics, International Research Centre,Kalasalingam Academy of Research and Education,
Krishnankoil-626126.Tamil Nadu, India.

devices [6-12].

Carbon spheres with metal hydroxides and metal oxides combination exhibits various extraordinary properties like high specific capacitance, surface areas, porous nature etc. which helps in enhancing their own property. Carbon spheres and cobalt hydroxide separately has unique properties, but on combining the both, it would further increase the physic-chemical, morphological and electrochemical property [13].

Supercapacitors have attracted greater interest as a best candidate for alternative energy resources. Although there is high progress in the electrochemical field, the developments in electrode fabrication with longer life cycle, efficiency, etc. is found to be a greater challenge for the scientific society. There are researchers working on all the three types of supercapacitors, such as electric double layer capacitors (EDLC), pseudocapacitors and hybrid capacitors [14-18].

In this present work, we have synthesized cobalt hydroxide layered carbon sphere via hydrothermal method. Herein, cobalt nitrate tetrahydrate and glucose has taken as precursor material and investigated the electrochemical behaviour of the prepared layered carbon sphere.

\section{MATERIAL SYNTHESIS}

\section{A. Chemicals}

Cobalt nitrate tetrahydrate and glucose from Sisco Research Laboratories Pvt. Ltd., Mumbai, India was used to synthesize the cobalt hydroxide layered carbon sphere. The analytical grade chemicals were used. $99 \%$ purity of all the chemicals were employed. Full reaction was carried out using De-ionized (DI) water as solvent. For electrochemical investigation $\mathrm{KOH}, \mathrm{PVDF}$, and NMP were brought from Sigma Aldrich Pvt. Ltd.

\section{B. Preparation process of cobalt hydroxide layered carbon sphere and modified working electrode fabrication}

The hydrothermal technique was used to prepare the cobalt hydroxide layered carbon sphere. $0.1 \mathrm{M}$ concentration of cobalt nitrate tetra hydrate was taken in $120 \mathrm{ml}$ DI water with stirring. Carbon sphere were prepared separately using $0.1 \mathrm{M}$ glucose. To the reaction mixture, the prepared carbon sphere was added and kept stirring. This reaction was transferred to autoclave and kept at $180^{\circ} \mathrm{C}$ for $12 \mathrm{~h}$. 
The centrifuging process was done to was out the unreacted materials with the help of DI water and ethanol for several times and the sample was dried at $60^{\circ} \mathrm{C}$ in oven for about $12 \mathrm{~h}$ [19].

Doctor blade technique was used to fabricate the cobalt layered carbon sphere modified working electrode.

\section{Material Characterization}

The structure of synthesized cobalt hydroxide layered carbon sphere crystal were analyzed using X-ray diffraction analysis (XRD) by Bruker X-ray diffractometer (D8 advance ECO) which has $\mathrm{Cu}-\mathrm{K}_{\alpha}$ radiation source and monochromatic wavelength range of $1.5406 \AA$. The functional group analysis was done by Fourier transform infrared spectrometer (FTIR) through Shimadzu (IR Tracer-100) spectrophotometer equipped within $4000-400 \mathrm{~cm}^{-1}$ range along with $\mathrm{KBr}$ pellet system. Scanning Electron Microscope (SEM) equipped with Energy Dispersive Spectroscopy (EDS) and mapping helped in analyzing the morphology, composition and distribution of elements by ZEISS-EVO 18 Research, Japan, and sample Electrochemical behavior was examined by electrochemical workstation which contained three electrode system $(\mathrm{CH}$ instrument 6008E).

\section{RESULTS AND DISCUSSION}

\section{A. XRD}

The XRD pattern of synthesized cobalt hydroxide layered carbon sphere was shown in Fig.1. The crystal structure formed was found to be in match with JCPDS card number 89-8616. The formation of nanomaterial was confirmed from the intense and broad peaks with sharp edges. The diffracted peaks with their corresponding hkl planes were similar to JCPDS number 89-8616. The synthesized cobalt hydroxide was found to be hexagonal structure [14]. The grain size of prepared nanomaterial was calculated from Scherrer formula provided in equation (1).

$$
D=k \lambda / \beta \operatorname{Cos} \theta \text {. }
$$

where $\beta$ is the Full-width Half Maximum (FWHM) and $\mathrm{k}$ is the constant. The calculated average crystallite size was 30 nm.

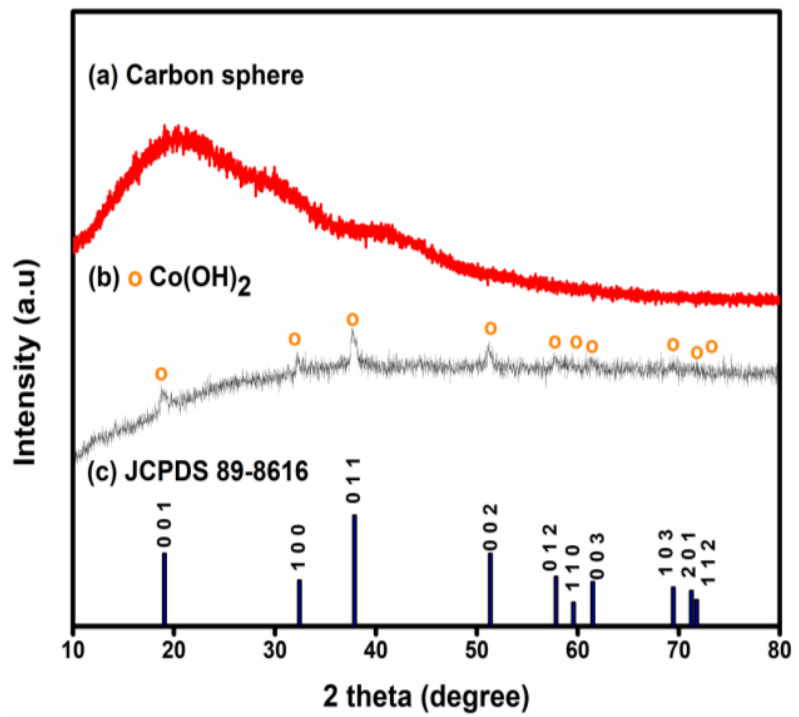

Fig. 1. XRD pattern (a) prepared bare carbon sphere (b) cobalt hydroxide layeredcarbon sphere (c) Jcpds date

\section{B. FTIR analysis}

The FTIR spectrum of prepared $\mathrm{Co}(\mathrm{OH})_{2}$ coated carbon spheres shown in Fig. 2. The spectral range was traced between 4000-400 $\mathrm{cm}^{-1}$. Bare carbon spheres and cobalt hydroxide coated carbon spheres was recorded. The small peaks were found at $569 \mathrm{~cm}^{-1}$ due to stretching vibration of $\mathrm{Co}-\mathrm{O}$ interaction. The strong peak near $1605 \mathrm{~cm}^{-1}$ indicated presence of $\mathrm{C}=\mathrm{O} . \mathrm{C} \equiv \mathrm{C}$ bond vibrations were observed in the peak $2824 \mathrm{~cm}^{-1}$. Medium stretching vibrations were found at 2857-2925 $\mathrm{cm}^{-1}$ corresponding to $\mathrm{CO}_{2}$ vibrations. The broad peak at $3203 \mathrm{~cm}^{-1}$ shows the $\mathrm{O}-\mathrm{H}$ bond formation which might be due to the absorption from the surrounding as it contains mesoporous structure [14, 19].

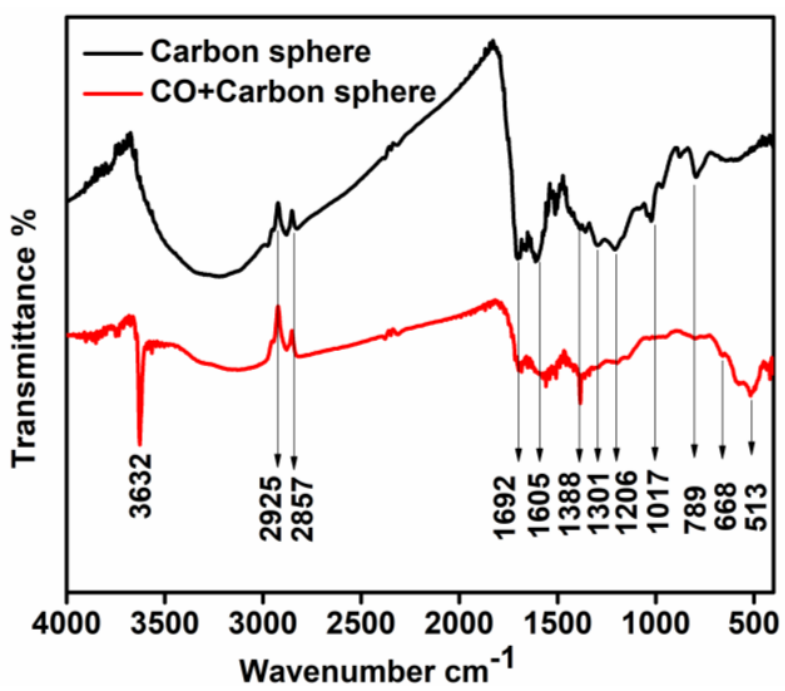

Fig. 2. FTIR spectrum of cobalt layered carbon sphere

\section{Surface and Elemental analysis}

Fig. 3. shows the SEM images of synthesized cobalt hydroxide layered carbon spheres.

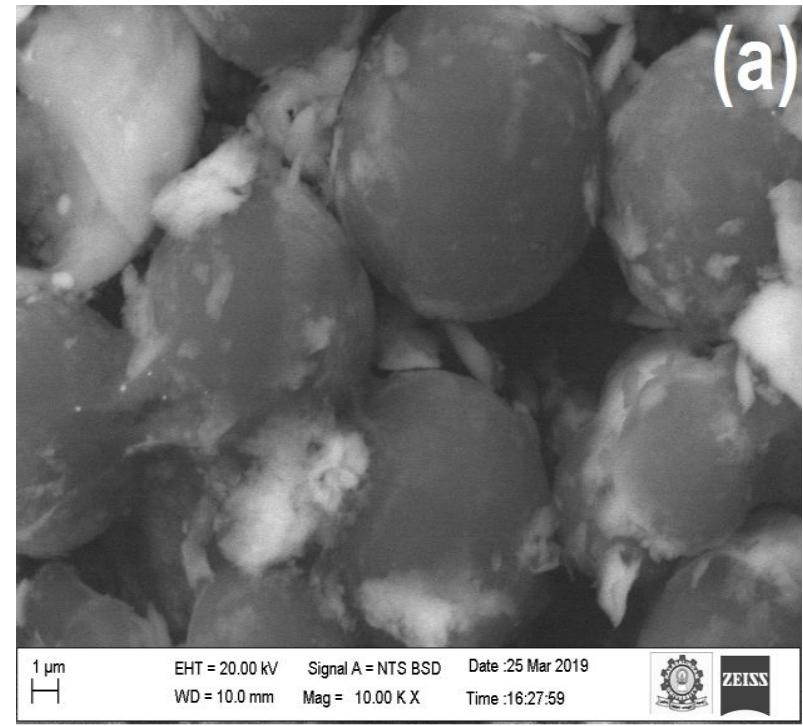

Fig. 3. (a) and (b) SEM images of cobalt hydroxide layered carbon sphere at $1 \mu \mathrm{m}$

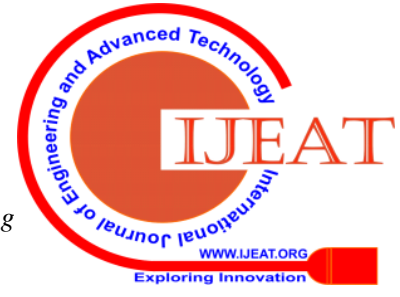


From the name it was clear that the prepared carbon sphere layered by cobalt hydroxide possessed sphere like morphology. The higher magnified images show the sphere shaped carbon sphere layered with cobalt hydroxide nanomaterials. In lower magnification, the layer formed above the carbon sphere surface was confirmed $[14,20]$.

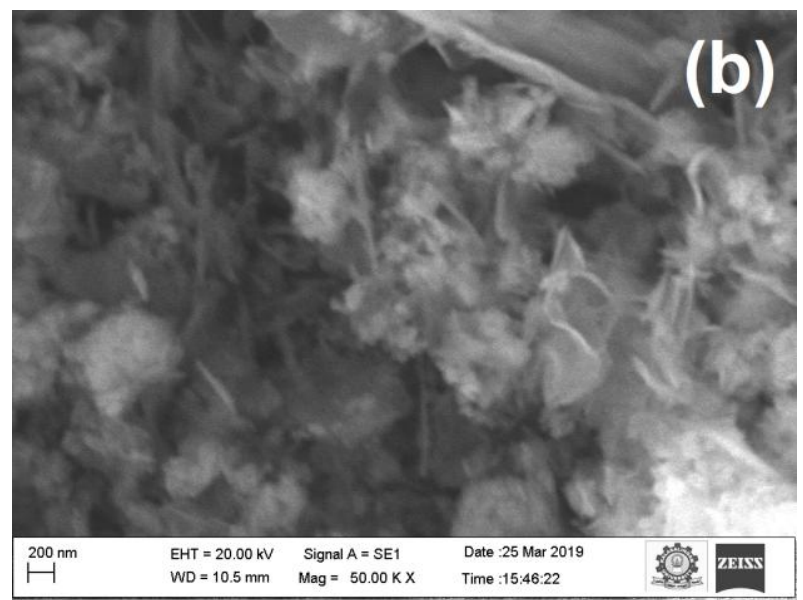

(b) Surface morphology of $\mathrm{CoOH}_{2}$ coated carbon sphere with $200 \mathrm{~nm}$

The fig 3(c) shows that the elemental composition of the prepared composite. The carbon contains the peak at the $\mathrm{K} \alpha$ radiation characteristic $\mathrm{X}$-ray emit at the range of $0.277 \mathrm{keV}$ and the intensity was high. It shows the moreover the percentage of carbon was very high from the EDAX graph $38.1 \%$. The Oxygen exhibits the range of radiation characteristic $\mathrm{x}$-ray $\mathrm{K} \alpha 0.525 \mathrm{keV}$ and the oxygen contain $33.5 \%$. Finally, the Ni peak contains in the range of $7.47 \mathrm{keV}$ in $28.4 \%$.

\section{EDS with mapping}

The purity of synthesized cobalt hydroxide layered carbon sphere was confirmed from the EDS spectrum. Fig. 4(a). shows the EDS spectrum and Fig. 4 (b-e) reveals mapping images of prepared cobalt hydroxide layered carbon sphere. The inset showed in Fig. 4 (a) provided the elemental weight percentage. The even distribution of cobalt hydroxide layered on to the carbon sphere was deduced.

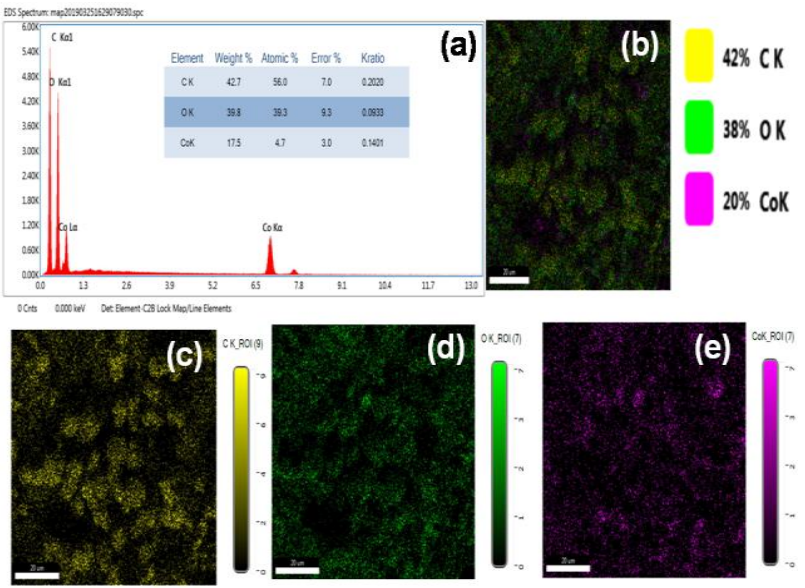

Fig. 4. (a) EDS spectrum and (b-e) mapping images of prepared cobalt hydroxide layered carbon sphere

\section{E. Electrochemical analysis \\ 1) Cyclic voltammetry}

The synthesized cobalt hydroxide layered carbon spheres were investigated for their redox property through cyclic voltammetry analysis. Fig.5. shows the $\mathrm{CV}$ graph with various scan rates of the synthesized cobalt hydroxide layered carbon sphere. Based on the redox curves potential window was adjusted from 0.2 to $0.6 \mathrm{~V}$. The oxidation and reduction peaks confirmed the pseudo capacitance behavior of synthesized cobalt hydroxide layered carbon spheres. The curves were found to be similar for all scan rates which shows that the prepared material had redox stability.

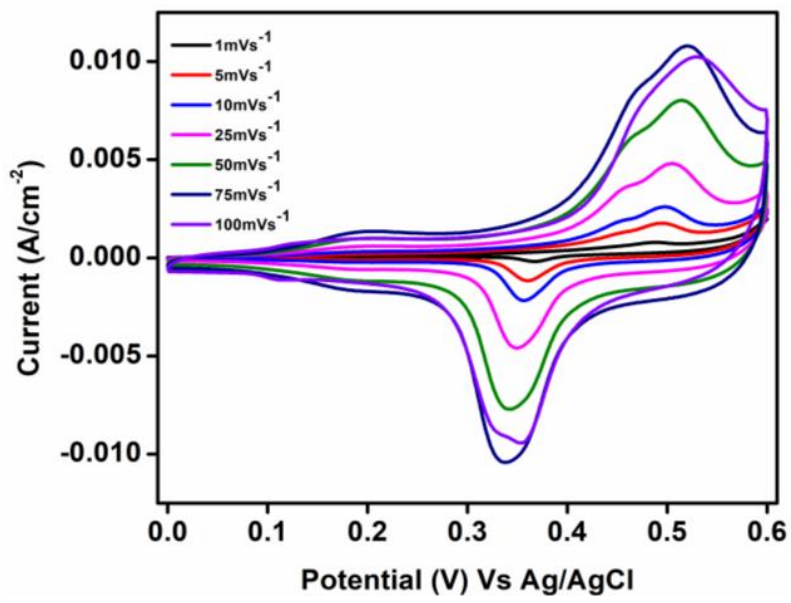

Fig. 5. CV graph of cobalt hydroxide layered carbon sphere

\section{2) Galvanostatic charge-discharge}

The Fig.6. shows the galvanostatic charge-discharge curve of synthesized cobalt hydroxide layered carbon sphere. A minimum current $0.5 \mathrm{~mA} / \mathrm{Cm}^{2}$ was applied to examine the charge discharge mechanism and potential window was fixed to 0 to $0.5 \mathrm{~V}$ in correspondence with the $\mathrm{CV}$ results. The discharge and charge curves were similar which shows the capacitance behavior of the prepared nanomaterials $[14,15$, 21].

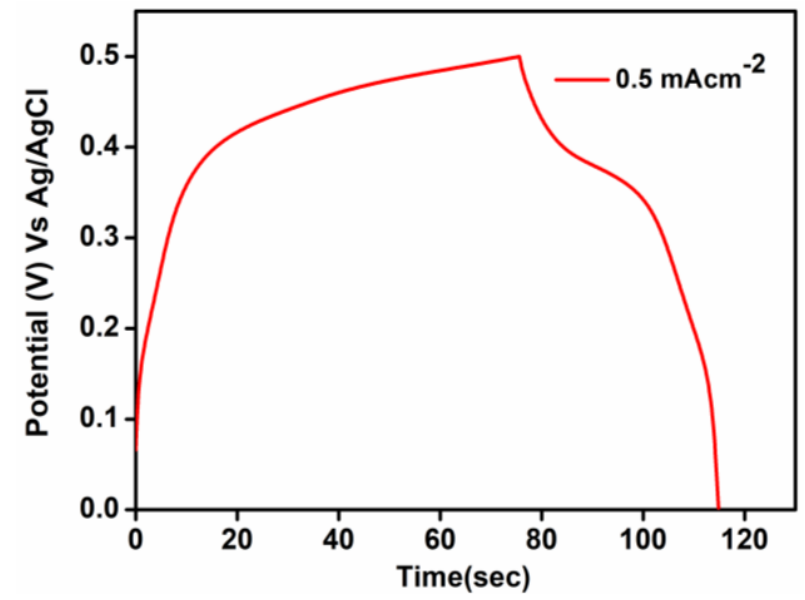

Fig. 6. The charge-discharge curve of $\mathrm{NiOH} 2$ coated carbon sphere

\section{CONCLUSION}

The cobalt hydroxide layered carbon sphere was synthesized by facile hydrothermal method. The hexagonal structure of synthesized cobalt hydroxide crystal along with carbon sphere was confirmed

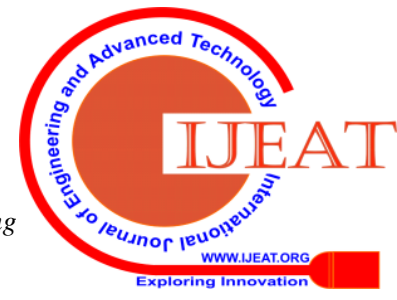


through XRD pattern. The bonding between carbon atoms and cobalt hydroxide were confirmed with the help of FTIR spectrum. EDS spectrum of the synthesized cobalt hydroxide carbon sphere were found to be in correspondence with the XRD result and mapping results. The weight percentage of atoms in EDS spectrum proved the purity through the absence of additional peaks and elemental compositions.

From the higher magnification SEM image, cobalt hydroxide layered on the outer surface of carbon sphere was proved. The oxidation and reduction peak formed in anodic and cathodic region implies pseudo capacitive nature of the synthesized cobalt hydroxide layered carbon sphere. Charge storage and discharge capability of the prepared nanomaterials were also confirmed which implies that the prepared nanomaterial was electrochemically active.

\section{ACKNOWLEDGMENT}

The authors feels thankful to IRC, Kalasalingam Academy Research and Education (KARE) for their University Research Fellowship (URF) and analyzation facilities supports.

\section{REFERENCES}

1. J-J. Chen, Q. Zhang, Y-N Shi, L-L Qin, Y. Cao, M-S. Zheng, Q-F. Dong, "A hierarchical architecture S/MW CNT nanomicrosphere with large pores for lithium sulfur batteries". Phys. Chem. Chem. Phys., 2012, 14, 5376-5382.

2. F. Wu, J. Li, Y. Su, J. Wang, W. Yang, N. Li, L. Chen, S. Chen, R. Chen, L. Bao, "Layer-by-layer assembled architecture of polyelectrolyte multi layers and graphene sheets on hollow carbon spheres/sulfur composite for high-performance lithium-sulfur batteries". Nano Lett. 2016, 16, 9, 5488-5494

3. N. Jha, P. Ramesh, E. Bekyarova, M. E. Itkis R. C. Haddon, "High Energy Density Supercapacitor Based on a Hybrid Carbon Nanotube-Reduced Graphite Oxide Architecture". Advanced Energy Materials, 2012, 2, 4, 438-444.

4. X. Fan, X. Chen, L. Dai, "3D graphene based materials for energy storage, Current Opinion in Colloid \& Interface Science”. Current Opinion in Colloid \& Interface Science 2015, 20, 429-438.

5. F. Liu, C. Wee Lee, Ji Sun Im, "Graphene-Based Carbon Materials for Electrochemical Energy Storage" Journal of Nanomaterials, 2013, $642915,1-11$

6. A. Shameem, P. Devendran, V. Siva, M. Raja, A. Manikandan, S. Asath Bahadur, "Preparation and Characterization of Nanostructured CdO thin films by SILAR method for Photocatalytic Application" Journal of inorganic and organometallic polymers and materials, 2017, 27, 692-699.

7. C. Zhang, Y. Li, D. Shuai, Y. Shen, W. Xiong, L. Wang, "Graphitic carbon nitride (g-C3N4)-based photocatalysts for water disinfection and microbial control: A review, Chemosphere, 2019, 214, 462-479.

8. R. Packiaraj, P. Devendran, S. Asath Bahadur, N. Nallamuthu, "Structural and electrochemical studies of Scheelite type BiVO4 nanoparticles: synthesis by simple hydrothermal method". J. of Materials Science: Materials in Electronics 2018, 29, 13265-13276.

9. P. Devendran, T. Alagesan, T. R. Ravindran, K. Pandian, "Synthesis of spherical CdS quantum dots using cadmium diethyldithiocarbamate as single source precursor in olive oil medium". Current Nanoscience, 2014, 10, 302-307.

10. K.Ravichandran, R. Mohan, B. Sakthivel, S. Varadharajaperumal, P. Devendran, T. Alagesan, K. Pandian, “Enhancing the photocatalytic efficiency of sprayed $\mathrm{ZnO}$ thin films through double doping $(\mathrm{Sn}+\mathrm{F})$ and annealing under different ambiences" Applied Surface Science, 2014, 321, 310-317.

11. P. Devendran, T. Alagesan, K. Pandian, "Single pot microwave synthesis of CdS nanoparticles in ionic liquid and their photocatalytic application" Asian Journal of Chemistry, Sup. Issue, 2013, 25, S79-S82.
12. M. Thiruppathi, P. Senthil Kumar, P. Devendran, C. Ramalingan, M. Swaminathan, E.R. Nagarajan, "Ce@TiO2 nanocomposites: An efficient, stable and affordable photocatalyst for the photodegradation of diclofenac sodium". Journal of Alloys and Compounds, 2018, 735, 728-734.

13. P. Devendran, T. Alagesan, A. Manikandan, S. Asath Bahadur, M. Krishna Kumar, S. Rathinavel, K. Pandian, "Sonochemical Synthesis of Bi2S3 Nanowires using Single Source Precursor and Study of Its Electrochemical Activity" Nanoscience and Nanotechnology Letters, 2016, 8, 1-6.

14. R. Packiaraj, P. Devendran, K. S. Venkatesh, S. Asath bahadur, A. Manikandan, N. Nallamuthu, "Electrochemical Investigations of Magnetic $\mathrm{Co} 3 \mathrm{O} 4$ Nanoparticles as an Active Electrode for Supercapacitor Applications". J. of Superconductivity and Novel Magnetism, 2018, 1-10, https://doi.org/10.1007/s10948-018-4963-6.

15. K. Seevakan, A. Manikandan, P. Devendran, A. Baykal, T. Alagesan, "Electrochemical and magneto-optical properties of cobalt molybdate nanocatalyst as high-performance supercapacitor". Ceramics International, 2018, 44, 17735-17742.

16. K. Seevakan, A. Manikandan, P. Devendran, A. Baykal, Y. Slimani, T. Alagesan "Structural, morphological and magneto-optical properties of $\mathrm{CuMoO}_{4}$ electrochemical nanocatalyst as supercapacitor electrode". Ceramics International 2018, 44, 20075-20083.

17. K. Seevakan, A. Manikandan, P. Devendran, A. Shameem, T. Alagesan, "Microwave combustion synthesis, magneto-optical and electrochemical properties of NiMoO4 nanoparticles for supercapacitor application". Ceramics International 2018, 44, 13879-13887.

18. R. Ranjithkumar, S. Ezhil Arasi, S. Sudhahar, N. Nallamuthu, P. Devendran, P. Lakshmanan, M. Krishna Kumar, "Enhanced electrochemical studies of $\mathrm{ZnO} / \mathrm{CNT}$ nanocomposite for supercapacitor devices”. Physica B: Condensed Matter 2019, 568, 51-59.

19. P. Devendran, T. Alagesan, K. Pandian, "Synthesis and characterization of $\mathrm{Bi}_{2} \mathrm{~S}_{3}$ nanorods decorated on carbon sphere and study its electrochemical application". Advanced Materials Research, 2014, 938, 215-220.

20. Q. Huang, K. Y. Liu, F. He, S-R. Zhang, Q-L. Xie, C. Chen, "Fabrication of cobalt aluminum-layered double hydroxide nanosheets/carbon spheres composite as novel electrode material for supercapacitors". Trans. Nonferrous Met. Soc. China 2017, 27, 1804-1814.

21. K. Seevakan, A. Manikandan, P. Devendran, Y. Slimani, A. Baykal, T Alagesan, "Structural, magnetic and electrochemical characterizations of $\mathrm{Bi} 2 \mathrm{Mo} 2 \mathrm{O} 9$ nanoparticle for supercapacitor application". Journal of Magnetism and Magnetic Materials 2019. doi: https://doi.org/10.1016/j.jmmm.2019.165254. 